\title{
DEMOCRACIA EM AÇÃO: indo além das eleições para melhorar o bem-estar ${ }^{1}$
}

\author{
Michael Touchton* \\ Natasha Borges Sugiyama * \\ Brian Wampler***
}

\begin{abstract}
Como a democracia contribui para melhorar o bem-estar? Neste artigo, separamos os componentes da prática democrática - eleições, participação social, expansão dos programas sociais, capacidade administrativa municipal - para identificar sua relação com o bem-estar. Nossa análise de um conjunto de dados original abrangendo mais de 5.550 municípios brasileiros demonstra que eleições competitivas sozinhas não explicam variação nas taxas de mortalidade infantil, um resultado associado ao bem-estar. Vamos além das eleições para mostrar como instituições participativas, programas sociais e capacidade administrativa municipal podem interagir para se apoiar e reduzir as taxas de mortalidade infantil. O resultado é uma nova compreensão de como os diferentes aspectos da democracia trabalham juntos para melhorar uma característica essencial do desenvolvimento humano.
\end{abstract}

Palavras-chave: Democracia, Bem-estar, Participação, Programas sociais, Brasil

\section{INTRODUÇÃO}

A democracia, de acordo com um volume significativo de estudos, contribui para o desenvolvimento humano por melhorar a vida dos cidadãos (Boix, 2001; Brown e Hunter, 2004; Gerring et al., 2015; McGuire, 2010). Evidências demonstram que, em média, países democráticos proporcionam mais bens públicos e padrões de vida mais elevados para seus cidadãos do que países autoritários (Besley e Kudamatsu, 2006; Lake e Baum, 2001;

* University of Miami. Department of Political Science. 1300 Campo Sano, Coral Gables, FL 33146. miketouchton@miami.edu

https://orcid.org/0000-0001-7645-1391

** University of Wisconsin, Milwaukee. Dept of Political Science, P.O. Box 413, Milwaukee, WI 53201. sugiyamn@ uwm.edu

https://orcid.org/0000-0002-5829-1512

$* * *$ Boise State University. 1910 University Dr. Boise, ID 83725, USA. bwampler@boisestate.edu

https://orcid.org/0000-0001-8338-8095

1 Publicado no American Political Science Review, 2017, Volume 111, Issue 1, p. 68-82. Permissão dos editores para publicar no Brasil. Esta é uma versão abreviada.

Tradução realizada por Ana Costa do artigo "Democracy at Work: moving beyond elections to improve well-being".
Przeworski et al., 2000). Mas como a prática democrática melhora a vida dos cidadãos? Defensores argumentam que eleições competitivas e a capacidade dos cidadãos de exercer direitos políticos contribuem para um governo receptivo e responsável. Os cidadãos exigem bens públicos e governos democráticos os providenciam, resultando, em média, em maior bem-estar (Rueschemeyer; Stephens, E; Stephens, J., 1992; Sen, 1999; Diamond,1999; Fox, 2015). No entanto, sistemas partidários fracos, conhecimento pouco aprofundado por parte dos eleitores, práticas clientelistas enraizadas, estados fragmentados e proteção apenas parcial dos direitos formalmente garantidos pelas constituições atingem muitas novas democracias (O’Donnell, 1998; Weyland, 1996; Ross, 2006; Cleary, 2007; Gibson, 2013).

Apesar desses desafios, algumas novas democracias estão melhorando e expandindo a provisão de bens públicos, o que aumenta as capacidades básicas e o bem-estar dos cidadãos (Sen, 1999; Gerring et al., 2015). Quando nos referirmos a bem-estar, nos inspiramos em Sen (1999), que define o termo como a "nossa capacidade de viver como gostaríamos" (Sen, 
1999, p. 13). Uma ampla variação no bem-estar entre democracias e dentro delas traz um enigma: Como a democracia promove o bem-estar? Abordar esse enigma é importante porque o bem-estar está ligado a muitos benefícios, incluindo aqueles associados a saúde, emprego, vida familiar e condições econômicas dos cidadãos. Por exemplo, estudiosos conectam níveis mais altos de bem-estar a um menor risco de doença, maior longevidade e maior produtividade econômica (Frey; Stutzer, 2002; Diener et al., 2009). Neste artigo, nos concentramos na mortalidade infantil como um indicador para o bem-estar, uma vez que a sobrevivência ao nascimento é uma pré-condição necessária para conduzir a vida escolhida (Nussbaum, 2011; Gerring et al., 2015).

Argumentamos que uma ampla compreensão da democracia permite uma melhor explicação para variações no bem-estar. ${ }^{2}$ Especificamente, fornecemos uma análise empírica robusta para mostrar como a introdução de uma extensa arquitetura de participação pública, a expansão do fornecimento de bens públicos e melhorias na capacidade estatal subnacional explicam a variação da mortalidade infantil no Brasil. Nossa contribuição teórica expande o cânone democrático para além das eleições, incluindo instituições participativas, programas sociais e capacidade estatal.

No restante deste artigo, nos baseamos primeiramente na teoria democrática para vincular uma conceituação mais ampla de democracia ao bem-estar. Em seguida, descrevemos as maneiras em que o Brasil funciona como um laboratório natural para examinar os vínculos entre a democracia e a taxa de mortalidade infantil, um indicador fundamental associado ao bem-estar. Posteriormente, descrevemos os dados e métodos que utilizamos para estimar esses relacionamentos. Por fim, analisamos

2 Neste artigo, nosso foco é a mortalidade infantil porque não temos espaço suficiente para examinar outras variáveis dependentes. Nós publicamos um livro em $2020 \mathrm{em}$ que examinamos quatro áreas incluindo saúde, educação, renda e gênero. Ver Wampler; Sugiyama; Touchton, 2020. os resultados da estimativa e discutimos suas implicações para a teoria e a prática democráticas.

\section{DEMOCRACIA EM AÇÃO}

Tal como a sua prática, a democracia é um conceito rico, confuso e altamente contestado. Muitos teóricos destacam o papel central da contestação, participação e cidadania como princípios fundamentais da democracia (Dahl, 1971; Pateman, 2012; Marshall, 1950). Dryzek nos lembra que a democracia é "dinâmica e aberta” (Dryzek, 2000, p. 29). Capturamos a complexidade da política democrática ao esclarecer como múltiplas características da democracia contribuem para o bem-estar. Grande parte da literatura empírica que avalia os efeitos da democracia se concentra em como as eleições influenciam o desempenho governamental e o bem-estar dos cidadãos (Gerring et al., 2015; Avelino; Brow; Hunter, 2005; Boix, 2001). Reconhecemos o papel das eleições na integração de preferências e na promoção da responsabilidade na tomada de decisões, mas concentramos nossa atenção teórica e empírica nas formas contínuas de participação cidadã, políticas sociais inclusivas e reforma do Estado como fatores cruciais para o bem-estar dos cidadãos.

Começamos com o papel da cidadania no projeto democrático devido à sua centralidade na teoria democrática (Dahl, 1971; Marshall, 1950; Somers, 2008). Nossa compreensão da cidadania democrática decorre das três dimensões complementares de Marshall: direitos civis, políticos e sociais. Garantir o acesso a esses direitos é fundamental para a política democrática, uma vez que os cidadãos devem ter amplas oportunidades para formular preferências, participar de ações individuais e coletivas, bem como de processos deliberativos em prol de seus interesses (Avritzer, 2002; Dahl, 1971; Dryzek, 2000; Fung; Wright, 2003).

Como as democracias podem construir 
uma cidadania mais substancial? Instituições democráticas criam oportunidades para que cidadãos coletem informações, se organizem e defendam seus interesses. A política eleitoral é uma possível via democrática porque permite aos cidadãos selecionar candidatos e partidos de acordo com as suas ideias de quem melhor representará seus interesses (Ferraz; Finan, 2011; Gerring et al., 2015; Huber; Stephens, 2012). O governo representativo fornece aos cidadãos mecanismos de responsabilização para encorajar autoridades eleitas a implementar políticas e programas de inclusão.

A participação, incluindo o diálogo contínuo e a deliberação entre autoridades governamentais, indivíduos e agentes organizados, representa outra via democrática para a cidadania. Essas formas de participação ocorrem em vários espaços, como audiências públicas, conferências sobre políticas públicas e manifestações de rua, tanto em níveis subnacionais como nacionais (Dryzek, 2000; Snyder, 2001; Tarrow, 1998). A responsabilidade social se expande quando os cidadãos e as organizações da sociedade civil (OSC) podem deliberar publicamente com funcionários do governo, propor políticas alternativas e monitorar a provisão de bens públicos (Fox, 2015; Putnam; Leonardi; Nanetti,1994; Perruzzoti; Smulovitz, 2000).

\section{Três percursos para o bem-estar}

Vamos além das eleições para conectar a democracia ao bem-estar mediante três percursos principais. Instituições participativas servem como um percurso ao promover a deliberação para a tomada de decisões que forjam novas relações - entre cidadãos, OSC e funcionários públicos - e estabelecem a base para o investimento em bens públicos necessários para cidadãos mais pobres. Instituições participativas são processos institucionais sancionados pelo Estado que transferem a autoridade decisória para espaços que incorporam funcionários públicos e cidadãos (Abers; Keck, 2013;
Avritzer, 2002; Baiocchi; Heller; Silva, 2011; Cornwall; Coelho, 2007; Fung; Wright, 2003; Wampler, 2015).

A riqueza - e a desordem - da política democrática está diretamente relacionada aos múltiplos espaços de competição por recursos escassos. Contestação política e competição permitem que cidadãos, grupos organizados e governos eleitos ampliem o acesso a direitos civis, políticos e sociais por meio do estabelecimento de novas instituições e políticas sociais (Marshall, 1950; Sen, 1999; Somers, 2008; Yashar, 2005). Pesquisas recentes sobre participação reconhecem uma gama mais ampla de processos e instituições, incluindo extensas experimentações com novas instituições democráticas e maior ênfase na transparência e no governo aberto (Fox, 2015; Pateman, 2012).

Políticas sociais destinadas a capacitar cidadãos representam o nosso segundo percurso para o bem-estar. O desenvolvimento, quando concebido através de uma abordagem baseada nas capacidades humanas, enfatiza "o papel crucial das oportunidades sociais na expansão da esfera da agência humana e da liberdade, tanto como um fim em si mesmo quanto como um meio de maior ampliação da liberdade” (Drèze; Sen, 2013, p. 8, tradução nossa). Pesquisas sobre democracias mais antigas revelam como o engajamento de cidadãos e uma ampla provisão social funcionam juntamente para criar efeitos de feedback político que reestruturam a política subsequente (Pierson, 1993).

Governos de países em desenvolvimento enfrentaram pressões duplas, buscando expandir a inclusão social enquanto encaravam restrições fiscais associadas às reformas econômicas neoliberais (Huber, 1996). Em vez de expandir o bem-estar social através de princípios universalistas, as novas democracias concentraram-se na inclusão social por meio de programas direcionados, visando maior eficiência e eficácia (Teichman, 2004). Programas de transferência condicionada de renda (TCR) exemplificam bem essa abordagem. Estudos 
sobre políticas sociais revelam que governos não precisam investir grandes somas de dinheiro para alcançar melhorias significativas no alívio da pobreza, na educação e na saúde. A formulação de políticas e o investimento progressivo em áreas que afetam os mais pobres, como escola primária e cuidados preventivos de saúde, são mais importantes do que os níveis absolutos de gastos (McGuire, 2010; Sugiyama, 2012).

A capacidade estatal nas democracias representa o nosso terceiro percurso para o bem-estar. Uma abordagem baseada nas capacidades humanas abre o escopo de ação para todos os cidadãos e enfatiza a capacidade estatal de fornecer bens públicos (Sen, 1999), em vez de focar na colaboração exclusiva com um grupo restrito de industriais em potencial para incentivar o investimento industrial (Kohli, 2004). Instituições democráticas podem apoiar uma abordagem baseada nas capacidades humanas de várias maneiras, tais como a expansão da sociedade civil através da proteção dos direitos civis e políticos, a ampliação dos pontos de acesso ao Estado e a expansão de direitos sociais (Evans; Heller, 2015; Ostrom, 1996; Sen, 1999). A capacidade do Estado de envolver os cidadãos e implementar reformas políticas está, portanto, intrinsecamente ligada a um processo democrático mais amplo de inclusão e participação.

O surgimento de instituições participatiจิ vas e programas sociais inovadores, bem como a necessidade de aproveitar a capacidade estatal local para prestar serviços, evidenciam a complexidade da prática democrática. Apesar dessa complexidade, cientistas políticos dedicaram sua atenção principalmente ao papel das eleições nacionais para explicar as conquistas democráticas relativas ao bem-estar (Gerring et al., 2015; McGuire, 2010). A maior parte do cânon democrático começa com o papel fundamental das eleições "livres e justas"; sem elas, a democracia se mostra ausente (Dahl, 1971). Do ponto de vista empírico, os dados eleitorais são publicamente disponíveis, mensuráveis e comparáveis, o que permite realizar pesquisas transnacionais. Infelizmente, o foco exclusivo nas eleições encobre as diversas maneiras pelas quais a contestação e a participação pública ajudam a construir a cidadania e a promover o envolvimento contínuo dos cidadãos nas atividades democráticas cotidianas. Esse enfoque também tem o potencial de apoiar-se demasiadamente em apenas uma instituição representativa, sugerindo que, se as eleições competitivas não produzirem melhores resultados para os cidadãos, a própria democracia pode estar em falta.

\section{A democracia em ação no Brasil}

Assim como outros países em desenvolvimento e de renda média, o Brasil experimentou altas taxas de mortalidade infantil no início do seu processo de democratização (WHO, 2015). É importante ressaltar que essas taxas caíram muito mais em algumas cidades e regiões do que em outras e que os governos subnacionais adotaram abordagens diferentes para implementar programas sociais (Brasil, 2016). Acadêmicos e profissionais do ramo ainda não sabem até que ponto novas instituições e eleições democráticas, programas sociais e ampliação da capacidade estatal são responsáveis pela redução da mortalidade infantil no Brasil, especialmente num contexto de crescimento econômico acelerado, que também deveria reduzir a mortalidade infantil. Explicar variações nesse indicador é essencial para entender o bem-estar de forma mais ampla.

Existe atualmente consenso sobre o impacto da mortalidade infantil no desenvolvimento (Nussbaum, 2011; Sen, 1999). Ela representa um indicador significativo do bem-estar, já que sobreviver além do primeiro ano de vida representa uma condição fundamental para a busca da vida desejada (McGuire, 2010; Nussbaum, 2011). O governo brasileiro coleta dados municipais sobre novas instituições democráticas, novos programas sociais, gestão orçamentária, eleições e mortalidade infantil. 
Esses permitem identificar possíveis vínculos entre essas áreas isoladas. Desse modo, o Brasil oferece uma oportunidade única para testar conexões entre instituições participativas, políticas públicas e aspectos administrativos da democracia com abrangência e profundidade nunca antes alcançadas.

\section{Instituições participativas}

A arquitetura participativa do Brasil expandiu-se nos anos 1990 (Avritzer, 2002; Baiocchi; Heller; Silva, 2011; Wampler, 2015). Conselhos de gestão de políticas públicas representam a instituição participativa mais comum no país, com quase 60 mil unidades e pelo menos 300 mil cidadãos eleitos para ocupar cargos nelas (Wampler, 2015, p. 264). O governo federal incentiva a adoção de conselhos de educação, saúde e assistência social por meio de incentivos fiscais ou controle regulatório. No entanto, existem pelo menos dezoito conselhos adicionais (como os de direitos das mulheres, segurança alimentar e direitos da criança), os quais são voluntários e não tão fortemente incentivados pelo governo federal (Gurza Lavalle; Voigt; Serafim, 2016). Análises extensivas de casos únicos e de pequenas amostras demonstram que as chances de adoção desses conselhos aumentam quando uma administração municipal reformista e uma sociedade civil ativa estão politicamente alinhadas (Almeida; Cayres; Tatagiba, 2015; Abers e Keck, 2013; Baiocchi; Heller; Silva, 2011; Wampler, 2015). A adoção desses conselhos pode ser liderada por prefeitos ou incentivada através de pressões estabelecidas por OSC. Nós enfatizamos aqui os conselhos de políticas públicas, em vez das conferências de políticas públicas e do orçamento participativo, porque os conselhos existem em todos os municípios brasileiros.

\section{Novos programas sociais}

As reformas do setor social brasileiro re- fletem a necessidade vivenciada em países em desenvolvimento de criar programas sociais voltados aos mais pobres. A partir de meados da década de 1990, as reformas sociais se espalharam por todo o país à medida que funcionários públicos e cidadãos desenvolveram soluções criativas para enfrentar problemas sociais de longa data (Sugiyama, 2012; Tendler, 1997). O Programa Saúde da Família (PSF), que hoje representa uma das principais estratégias de atenção à saúde do Sistema Único de Saúde (SUS), fornece saúde básica, concentrando-se em cuidados preventivos definidos geograficamente por meio de uma equipe de profissionais de saúde (Viana; Dal Poz, 1998).

O Bolsa Família foi criado em 2003 e representa o maior programa de TCR do mundo. É a ação de alívio da pobreza mais visível e abrangente do governo, incluindo cerca de um quarto da população. Apesar das condições de acesso ao benefício, o discurso do governo enfatiza uma perspectiva baseada em direitos para a inclusão de beneficiários ao programa (Hunter; Sugiyama, 2014). Assim como o PSF, o Bolsa Família contribuiu para o desenvolvimento humano, principalmente em áreas relacionadas a educação e saúde (Rasella et al., 2013; Soares; Ribas; Osório, 2010).

\section{Capacidade estatal}

Os municípios brasileiros apresentam desenvolvimento econômico desigual e experiências variadas com clientelismo (Ames, 2001; Hagopian, 1996). A governança descentralizada, portanto, apresenta sérios desafios para a entrega padronizada e universal de benefícios sociais. Primeiramente, a Lei de Responsabilidade Fiscal, promulgada em 2000, ampliou a transparência fiscal e implementou novas regras sobre gastos públicos. Com essa lei, governos locais devem investir 50\% de seu orçamento anual em saúde e educação e reportar dados fiscais às autoridades federais anualmente. Em segundo lugar, o estabelecimento do Tribunal de Contas da União e da 
Controladoria-Geral da União garantiu o monitoramento contínuo da implementação de políticas públicas (Speck, 2011). Finalmente, inovações nas práticas de gestão ministerial, como as do Ministério do Desenvolvimento Social (MDS), conectaram a supervisão administrativa da gestão local a incentivos financeiros para o "bom desempenho" (Lindert et al., 2007). Juntas, a incorporação da nova gestão pública (Grindle, 2007) e as garantias constitucionais representam uma reformulação da provisão social em termos de direitos sociais e acesso ao Estado (Hunter; Sugiyama, 2014).

\section{Efeitos interativos}

Instituições participativas, novos programas sociais e capacidade estatal interagem entre si para explicar a variações no bem-estar. Primeiramente, instituições participativas influenciam o desempenho de programas sociais e a capacidade do governo local de prestar serviços, ao expandir o debate sobre políticas públicas, permitindo que os cidadãos proponham novas políticas e se envolvam na supervisão da implementação de novos projetos (Baiocchi; Heller; Silva, 2011; Pogrebinschi; Samuels, 2014). Espaços participativos também permitem que os cidadãos tragam conhecimentos locais para lidar com discussões políticas complexas (Wampler, 2015). Em segundo lugar, instituições participativas servem como um hub que liga o Estado à sociedade e um fórum para disseminar informações sobre limitações na gestão de programas sociais (Abers; Keck, 2013). Os conselhos de políticas públicas fazem parte de uma nova teia de "instituições entrelaçadas", através da qual instituições participativas, departamentos governamentais e outros órgãos do governo interagem entre si e se fortalecem por meio de conexões formais e informais (Wampler, 2015). Uma vez conscientes dos problemas existentes, os membros dos conselhos podem aumentar a pressão sobre as autoridades locais para melhorar a prestação de serviços (Fox, 2015).
Finalmente, a capacidade estatal local melhora o desempenho de instituições participativas e programas sociais. A capacidade da administração local de implementar políticas e se envolver com conselhos de políticas públicas é fundamental para a credibilidade e sustentabilidade desses conselhos, uma vez que eles têm menor influência se as políticas escolhidas por eles nunca forem implementadas. Além disso, um governo local mais capaz pode fornecer melhores informações para os processos de deliberação e sua capacidade de administrar programas sociais federais está diretamente relacionada à capacidade local e à adesão a metas programáticas. A crença de que o acesso a bens públicos é um direito social, e não um favor pessoal, precisa ser reforçada por meio de políticas estruturadas na esfera federal e implementadas localmente (Sugiyama; Hunter, 2013). A provisão tecnocrática de serviços sociais garantidos constitucionalmente tem o poder de elevar os padrões de vida dos cidadãos mais pobres e transformá-los em cidadãos plenos.

\section{O papel do crescimento econômico}

Desde a redemocratização, o Brasil passou por períodos de declínio econômico e de crescimento. Em meados da década de 1990, as reformas neoliberais resultaram numa redução do Estado. Um período de rápido crescimento econômico ocorreu durante os anos 2000, como parte do boom das commodities e do retorno de um Estado neodesenvolvimentista. Esse crescimento possibilitou uma infusão de renda nas famílias mais pobres e trouxe novo capital para o país. Para os brasileiros mais pobres, os anos 2000 trouxeram a mais significativa oportunidade para ascensão social em gerações (Ipea, 2016). As conexões possíveis entre crescimento econômico e melhorias no bem-estar ocuparam o centro dos debates recentres sobre economia (Dollar; Kraay, 2001; Rodrik, 2000). Alguns estudos vinculam aumentos no crescimento a reduções na pobreza 
(Dollar; Kraay, 2001; Ferreira, 2010), enquanto outros concluem que o crescimento tem pouco impacto sobre a pobreza (Xue, 2012).

Muitos estudos nessa área são incapazes de explicar variáveis de confusão em escala global. No entanto, algumas pesquisas se concentram em um subconjunto de países ou em um país em particular para resolver esse problema (Ravallion; Chen, 2007). Nosso foco no Brasil segue essa abordagem. Incluímos variáveis de controle econômico local em nossos modelos estatísticos de mortalidade infantil porque outros autores também conectam o crescimento econômico local à pobreza e ao bem-estar no Brasil (Lustig; Lopez-Calva; Ortiz-Juarez, 2013; McGuire, 2010).

\section{Metodologia: projeto de pesquisa e sele- ção de caso}

Com base em um conjunto original de dados que abrange 5.570 municípios brasileiros, avaliamos conexões entre instituições locais participativas, programas sociais federais e mortalidade infantil. Concentramo-nos na mortalidade infantil como um indicador do bem-estar porque a sobrevivência ao nascimento representa uma condição necessária para o conforto, a saúde e a felicidade enquanto buscamos a vida desejada (McGuire, 2010; Nussbaum, 2011). Além disso, a mortalidade infantil é um indicador útil para testar nossos argumentos, uma vez que pode mudar rapidamente devido a intervenções políticas e negligência (Aquino; Oliviera; Barreto, 2009).

Variável dependente: óbitos infantis a cada mil nascidos vivos

Utilizamos a estimativa do Ministério da Saúde relativa ao índice anual de mortalidade infantil a cada mil nascidos vivos em cada município brasileiro entre 2006 e 2013. A média é de 21,14 com um desvio padrão de 22,21. Fonte: www.datasus.gov.br

\section{Variáveis independentes}

Adoção de conselhos de políticas públicas relacionados à saúde

Utilizamos os dados de uma pesquisa do Instituto Brasileiro de Geografia e Estatística (IBGE, 2013) sobre a presença de 21 diferentes conselhos de políticas públicas nos municípios brasileiros. Dentre eles estão conselhos de saúde, direitos da mulher, habitação, cultura, entre outros. Muitos municípios adotam alguns, como conselhos de saúde e educação, devido a incentivos financeiros federais. Por exemplo, a taxa de adoção média do conselho de saúde em nossos dados é $80 \%$, atingindo quase $100 \%$ em 2013. Desse modo, a presença de um conselho de saúde é uma medida inadequada para testar nossos argumentos devido a essa baixa variação. No entanto, é possível que outros conselhos influenciem os níveis mortalidade infantil, já que esse problema afeta desproporcionalmente cidadãos pobres, mulheres, afro-brasileiros, povos indígenas e crianças. Seis dos conselhos em nosso conjunto de dados (direitos da mulher, direitos da criança, segurança alimentar, saneamento, saúde da mulher e política urbana) tratam de certas causas da mortalidade infantil, o que significa que os participantes deliberam sobre questões que afetam diretamente a mortalidade infantil.

A presença desses seis conselhos também permite um teste mais preciso dos nossos argumentos do que a presença de conselho de saúde, porque a deles não é uma exigência. Tratamos os que não recebem nenhum financiamento federal como sendo mais "voluntários" do que aqueles para os quais há claro benefício financeiro de incentivo à adoção municipal. Também supomos que a adoção desses conselhos mais voluntários representa maior comprometimento da sociedade civil e da administração municipal com a participação democrática do que a adoção de conselhos com incentivos federais (Pires; Vaz, 2012; Gurza Lavalle; Voigt; Serafim, 2016). 
Duas variáveis dummy incorporam os conselhos de políticas locais. A primeira é codificada como "1" se um município apresentar todos os seis conselhos voluntários que poderiam estar relacionados à mortalidade infantil em determinado ano, e "0" se esse não for o caso. A segunda variável registra se os municípios têm os conselhos que possuem fundos federais e que podem influenciar aspectos vinculados à saúde. Dentre eles estão os conselhos de saúde, habitação, educação e meio ambiente. Sessenta e três por cento das observações municipais apresentam esses quatro conselhos, sendo codificados como " 1 "; o restante é codificado como " 0 ". Finalmente, registramos a frequência das reunióes do conselho de saúde. Muitos municípios adotam conselhos de saúde para obter fundos federais, mas, uma vez adotados, esses conselhos podem receber pouco incentivo para serem ativos e se sustentarem ao longo do tempo. Buscamos capturar a variação no compromisso relativo ao uso desses conselhos através do número de reuniões que eles realizam anualmente. O número médio de reuniões é de 10,4 e o desvio padrão é 5,4.

\section{Cobertura do Bolsa Família}

Nossos modelos incluem um indicador para a cobertura municipal do Bolsa Família: o percentual de famílias elegíveis que recebem benefícios do programa ${ }^{3}$. O nível médio de cobertura é 83\% em nossos dados e o desvio padrão é 31. Esta é a mesma variável usada em Rasella et al. (2013) e Macinko; Guanais e Souza (2006).

Cobertura do Programa Saúde da Família (PSF)

Incorporamos aos nossos modelos de mortalidade infantil dados do Ministério da Saúde relativos à cobertura municipal do PSF. O Ministério coleta dados anuais sobre a por-

3 www.mds.gov.br/assuntos/bolsa-familia centagem de famílias elegíveis que recebem os benefícios do programa. O nível médio de cobertura é de 81\% e o desvio padrão é de 29.

\section{Qualidade da Gestão do Bolsa Família}

O programa Bolsa Família é administrado em nível municipal e sua qualidade administrativa varia consideravelmente ao redor do país. Utilizamos dados operacionais do MDS, conhecidos como Índice de Gestão Descentralizada (IGD), para capturar essa variação. O MDS oferece maior financiamento para as cidades que apresentam melhor desempenho no IGD. Esta variável é contínua de 0 a 1 e cada município recebe uma pontuação anual. Uma melhor gestão receberá pontuações mais próximas de 1 e um pior gerenciamento será colocado próximo de zero. A pontuação média é de 0,76 e o desvio padrão é de 0,15.

\section{Gastos municipais per capita com saúde}

Nós avaliamos até que ponto os gastos públicos contribuem para o bem-estar. O Brasil gasta um nível comparativamente alto de recursos na provisão de bens públicos, mas não atinge resultados consistentes de alta qualidade nos indicadores de desenvolvimento humano (McGuire, 2010; Sugiyama, 2012). Crescimento econômico pode gerar um aumento na receita local que facilite o crescimento dos gastos com assistência médica. Incluímos um indicador de investimentos per capita em saúde para capturar essa possibilidade e explicar a variação dos gastos entre as cidades brasileiras. Essa medida também nos permite contabilizar aumentos na receita e nos gastos ao longo do período incluído neste estudo. Esses valores estão em reais constantes e vêm do Ministério da Saúde.

\section{Salários de baixa renda}

Tendências econômicas também podem influenciar as taxas de mortalidade infantil 
de diversas formas no âmbito individual. Primeiramente, desnutrição e deficiências no saneamento básico são as principais causas de mortalidade infantil (Rasella et al., 2013). Uma renda maior expande o acesso dos cidadãos a água limpa e comida. Assim, salários mais altos entre cidadãos de baixa renda estão associados a níveis mais baixos de mortalidade infantil. Em segundo lugar, os salários dos cidadãos influenciam a sua capacidade de comprar bens privados. $\mathrm{O}$ acesso a cuidados de saúde de qualidade através do sistema universal de saúde é desigual; empregadores do setor formal e cidadãos de renda média ou alta muitas vezes contam com seguros de saúde particulares. Desse modo, levantamos a hipótese de que o risco de mortalidade infantil diminui à medida que os salários aumentam. Para incorporar essa perspectiva, incluímos em nossos modelos uma variável de salários municipais médios para o quintil mais baixo de pessoas assalariadas. Esses valores estão em reais constantes e vêm do IBGE.

\section{Eleições competitivas}

Codificamos de várias maneiras os dados sobre a competitividade relativa das eleições municipais. Primeiramente, codificamos uma variável dummy com uma pontuação de "1" se o candidato a prefeito vencedor recebeu menos de $45 \%$ no primeiro turno e " 0 " se recebeu mais de $45 \%$. O recebimento de menos de $45 \%$ dos votos no primeiro turno demonstra um ambiente eleitoral competitivo em que o prefeito pode ter que se preocupar com a possibilidade de reeleição. Finalmente, criamos uma variável dummy para registrar se os prefeitos tiveram oposição na eleição anterior (“1” indica prefeito sem oposição). Esses valores são do Tribunal Superior Eleitoral: http:// www.tse.jus.br/.

Prefeitos do PT e de esquerda

Estudos anteriores conectaram prefeitos do Partido dos Trabalhadores (PT) a baixos níveis de mortalidade infantil (Touchton; Wampler, 2014). Prefeitos do PT têm historicamente apoiado a participação política local. Da mesma forma, pesquisas subnacionais sobre reformas de saúde e educação concluem que partidos de esquerda e de centro-esquerda são mais propensos a adotar políticas sociais progressistas (Sugiyama, 2012). Para incorporar essa perspectiva, codificamos uma variável dummy como " 1 " se os municípios tiverem um prefeito do PT em um determinado ano, com os prefeitos de todos os outros partidos recebendo uma pontuação de " 0 ".

\section{Eleições presidenciais}

Incluímos uma variável de apoio municipal aos candidatos presidenciais do PT eleitos em 2002, 2006 e 2010. Esses presidentes promoveram instituições participativas e programas sociais. Buscamos controlar a possibilidade de que alguns municípios e seus residentes estejam mais comprometidos com programas e instituições associados a esses presidentes.

\section{RESULTADOS E DISCUSSÃO}

Tabela I. Variações na mortalidade infantil, 20062013. Este modelo utiliza uma série temporal binomial negativa dwe efeitos minicupais fixos.

\begin{tabular}{l|cc}
\hline \multicolumn{1}{c|}{ Variável } & $\begin{array}{c}\text { Coeficiente } \\
(\mathrm{EP})\end{array}$ & $\begin{array}{c}\text { Coeficiente } \\
\text { (EP) }\end{array}$ \\
\hline $\begin{array}{l}\text { Comprometimento de } \\
\text { conselhos voluntários }\end{array}$ & $-0,21^{* *}(0,03)$ & \\
$\begin{array}{l}\text { Frequência de reuniões do } \\
\text { conselho de saúde }\end{array}$ & & $0,001(0,003)$ \\
& & \\
Cobertura do PSF & $-0,002^{* *}(0,0001)$ & $-0,002^{* *}(0,0002)$ \\
Cobertura do Bolsa Família & $-0,0005^{*}(0,0008)$ & $-0,0004^{*}(0,0002)$ \\
& & $-0,24^{* *}(0,03)$ \\
Gestão do Bolsa Família & $-0,22^{* *}(0,03)$ & $-0,0009$ \\
& $-0,001$ & $(0,003)$ \\
Competição para Prefeitura & $(0,003)$ &
\end{tabular}




\begin{tabular}{l|cc} 
Prefeito do PT & $-0,02(0,02)$ & $-0,05(0,11)$ \\
Eleição Presidencial & 0,002 & $-0,001$ \\
& $(0,001)$ & $(0,003)$ \\
Gastos Per Capita em Saúde & $-0,00002$ & $-0,00002$ \\
& $(0,00002)$ & $(0,00004)$ \\
Salários de Baixa Renda & $0,0006^{*}$ & $0,0006^{* *}$ \\
& $(0,0002)$ & $(0,0002)$ \\
Constante & $2,15^{* *}(0,07)$ & $2,03^{* *}(0,07)$ \\
\hline N & 20,199 & 20,260 \\
Wald Chi2 & 255,91 & 199,69 \\
Prob> Chi2 & 0,000 & 0,000 \\
\hline
\end{tabular}

* indica significância melhor que 0,05 (teste bicaudal).

* * indica significância melhor que 0,01 (teste bicaudal).

Fonte: Elaborada pelos autores.

O modelo da Tabela I apresenta várias constatações importantes. Em primeiro lugar, os conselhos voluntários de políticas públicas têm uma conexão forte, negativa e estatisticamente significativa com a mortalidade infantil no Brasil. Estimamos que a presença desses conselhos voluntários esteja associada a uma redução de 0,21 na mortalidade infantil a cada mil nascimentos. Essa redução pode parecer pouco significativa com relação à taxa de mortalidade infantil média de 21,15 a cada mil nascimentos, mas os efeitos são importantes. Esses coeficientes refletem a diferença nos registros das contagens esperadas do indicador de mortalidade infantil. Um coeficiente de 0,21 significa uma redução de 1,62 na mortalidade infantil a cada mil nascimentos, repre: sentando uma redução de 14\% em relação às taxas médias atuais. Em média, estimamos que os conselhos voluntários relacionados à assistência médica preservem aproximadamente 380 vidas por ano no Brasil e mais de 3 mil para os anos incluídos em nosso estudo. Por extensão, nossos resultados destacam a importância do comprometimento com instituições participativas locais para avançar o bem-estar.

Utilizamos uma variável com a frequência das reuniões do conselho de saúde no segundo modelo da Tabela I, porque muitos municípios podem aceitar fundos federais para a criação do conselho, mas isso não garante que ele se reúna regularmente. O segundo conjunto de resultados da Tabela I mostra qwue não há relação entre os municípios com conselhos de saúde que se reúnem mais de uma vez por mês e a mortalidade infantil. Existem várias razões possíveis para esses resultados. A primeira é que instituições impostas de cima para baixo tendem a não ter um bom desempenho em comparação com aquelas adotadas de baixo para cima, como sugerem pesquisas anteriores sobre inovações democráticas (McNulty, 2011). A segunda é que a nossa medida de frequência das reuniões pode não capturar o comprometimento de um município com instituições participativas. Quase todos os municípios brasileiros têm conselhos de saúde e a realização de mais reuniões nesses conselhos pode não indicar progresso em direção a reformas políticas ou à prestação de serviços de saúde. Finalmente, nosso indicador para as reuniões do conselho de saúde contém variação consideravelmente menor do que para os conselhos voluntários: mais da metade dos conselhos de saúde induzidos se reúne mensalmente ou bimestralmente. Portanto, não encontramos apoio para os nossos argumentos ao utilizar a frequência de reuniões do conselho de saúde como uma variável independente, mas para outros estudos em instituições participativas induzidas de cima para baixo (McNulty, 2011).

\section{Programas sociais federais}

Nossa segunda constatação mostra a importância de programas sociais federais estabelecidos de cima para baixo, representando abordagens guiadas por especialistas para a prestação de serviços. Uma maior abrangência dos programas Bolsa Família e Saúde da Família está associada a níveis mais baixos de mortalidade infantil. Esses resultados reproduzem descobertas proeminentes da literatura voltada a ambos os programas, como os estudos de Rasella et al. (2013) e Macinko; Guanais e Souza (2006). É importante ressaltar também 
a importância da gestão local do Bolsa Família. O indicador federal para a qualidade da gestão local do programa, IGD, também é um determinante estatisticamente significativo das taxas de mortalidade infantil. Estimamos que o aumento do índice médio de gestão municipal $(0,76)$ para dois desvios padrão acima da média $(0,99)$ resultará em uma importante redução na mortalidade infantil.

É importante mencionar que esses resultados controlam os gastos municipais per capita em saúde e os salários de baixa renda. Como os gastos com saúde não se mostram relacionados à mortalidade infantil em nossos dados, reduzir a mortalidade infantil, como defendido por McGuire (2010), não é simplesmente uma questão de quanto os municípios gastam em saúde, mas também de como investem seus próprios recursos (conforme monitorado pelos conselhos de políticas públicas) e os recursos federais destinados ao Bolsa Família (como evidenciado pelos índices de gestão). Surpreendentemente, os salários de baixa renda têm uma relação positiva e estatisticamente significativa com a mortalidade infantil. Isso pode significar que salários mais altos no quintil mais baixo de assalariados em regiões agrárias produtoras de commodities tendem a ser mal governados.

\section{Eleições}

Encontramos baixo consenso quanto a conexões entre eleições competitivas e mortalidade infantil em nossos dados. Primeiramente, não identificamos relação entre eleições competitivas e mortalidade infantil quando usada a variável dummy. Descobrimos que os prefeitos que concorrem sem oposição estão associados a níveis sistematicamente mais altos de mortalidade infantil, o que corrobora os argumentos que associam uma total falta de competição política com clientelismo e fraco desempenho governamental. Apesar dos resultados mistos para variáveis eleitorais, nossos modelos não contrariam estudos anteriores so- bre eleições, democracia e bem-estar em um contexto transnacional. Em vez disso, nossos resultados destacam a importância da complexidade democrática para além das eleições.

Finalmente, nossos resultados mostram que a influência mais significativa sobre a mortalidade infantil se deve à interação contínua de três componentes principais - a presença de instituições participativas ativas, programas sociais inovadores e um governo municipal mais capaz. Os resultados da Tabela II mostram como a interação entre os conselhos voluntários de políticas públicas e a cobertura do PSF está associada aos níveis de mortalidade infantil em nossos dados. Municípios no primeiro quintil de cobertura do PSF e com um comprometimento com os conselhos voluntários relacionados à assistência à saúde estão vinculados a uma redução de 1,17 na taxa de mortalidade infantil anual a cada mil nascimentos. Isso corresponde a uma redução média estimada na mortalidade infantil de $15 \%$, mas que pode chegar a $30 \%$ em alguns municípios - traduzindo-se em cerca de 1.400 vidas salvas por ano nos municípios em nosso conjunto de dados, e 14.000 ao longo de todo o período abrangido em nosso estudo. Assim, o PSF funciona melhor quando a administração local é capaz de registrar corretamente famílias elegíveis e garantir que os beneficiários sejam incorporados a uma rede maior de programas de apoio, como outros conselhos de políticas públicas.

Existem muitas diferenças entre os municípios brasileiros, além da presença de conselhos locais e cobertura do PSF. No entanto, suponhamos que todos tenham mostrado um comprometimento com instituições participativas e ampla cobertura para o PSF, mantendo todo o resto constante. A estimativa total de vidas que seriam salvas ao longo do período do nosso estudo, se todos os municípios estivessem nessa situação, é de 112 mil, ou aproximadamente $22 \%$ do total de óbitos infantis entre 2006 e 2013. 
Tabela II. Variações na mortalidade infantil, 2006- amostra em municípios com baixo, médio e 2013. Esses modelos utilizam um termo de intera- alto nível de mortalidade infantil para abordar ção e diferentes configurações de conselhos de polí- o potencial para relações não lineares. Nossos ticas e cobertura do programa de Saúde da Família. resultados são robustos para estas especifica-

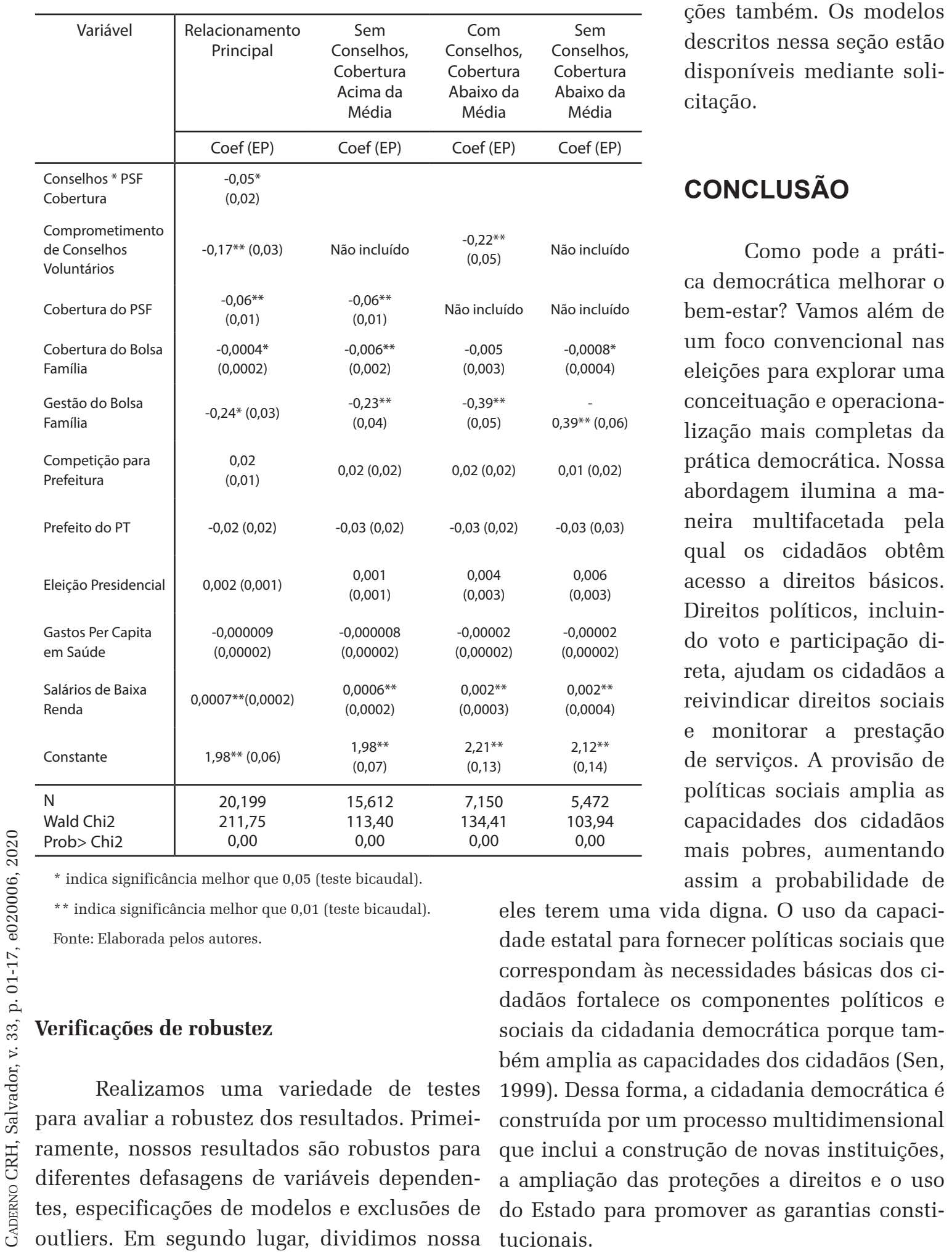


Nosso vasto conjunto original de dados nos permite estabelecer novos campos empíricos e ir além das eleições e do crescimento econômico para conectar a democracia ao bem-estar. Primeiramente, descobrimos que a presença de instituições participativas está associada a redução da mortalidade infantil. Este é o primeiro estudo a demonstrar quantitativamente uma relação forte e positiva entre instituições participativas e bem-estar em um número tão grande de casos. A segunda constatação empírica é que políticas sociais inovadoras contribuem para melhorias nos indicadores na mortalidade infantil. Por fim, descobrimos que uma maior capacidade estatal aplicada à provisão social também contribui para melhorias no bem-estar. Pesquisas anteriores examinaram cada fator isoladamente, enquanto nós demonstramos como essas práticas funcionam de forma independente e interativa para explicar a variação na mortalidade infantil, que é menor nos municípios onde há maior comprometimento com instituições participativas e com políticas sociais que expandem os direitos básicos. Esses resultados são importantes porque sugerem que instituições participativas, programas sociais e capacidade local se reforçam mutuamente para melhorar o bem-estar. O alto desempenho em um aspecto da democracia pode reduzir a mortalidade infantil, mas uma constelação de práticas e programas democráticos, junto a um Estado capaz funciona melhor.

Num sentido mais abrangente, esses indícios sugerem que os defensores da democracia devem usar uma abordagem multidimensional para ativar a cidadania e melhorar o bem-estar (Ver Wampler; Sugiyama; Touchton, 2020). Eleições livres e justas representam um mecanismo importante para que os cidadãos alcancem o bem-estar, mas não necessariamente o mais importante. A política pública e o desenvolvimento institucional, incluindo novas instituições democráticas, políticas sociais inovadoras e capacidade estatal democrática, também são centrais para os esforços de redução da mortalidade infantil. Desta forma, as democracias podem ir além das eleições para melhorar o bem-estar.

Recebido para publicação em 25 de agosto de 2019 Aceito em 07 de maio de 2020

\section{REFERÊNCIAS}

ABERS, Rebecca; KECK, Margaret. Practical Authority. Oxford: Oxford University Press, 2013.

ALMEIDA, Carla, CAYRES, Domitila Costa; TATAGIBA, Luciana. "Balanço dos Estudos Sobre os Conselhos de Políticas Públicas na Última Década.” Lua Nova 94 (April) 255-294, 2015.

AMES, Barry. The Deadlock of Democracy in Brazil. Ann Arbor: University of Michigan Press, 2001.

AQUINO, Rosana; OLIVIERA, Nelson F. de.; BARRETO, Mauricio. "Impact of the family health program on infant mortality in Brazilian municipalities." American Journal of Public Health 99:1 (January): 87-93, 2009.

AVELINO, George, BROW, David ; HUNTER, Wendy. Hunter. "The effects of capital mobility, trade openness, and democracy on social spending in Latin America, 1980-1999.” American Journal of Political Science 49:3 (July), p. 625-641, 2005.

AVRITZER, Leonardo. Democracy and the Public Space in Latin America. Princeton: Princeton University Press, 2002.

BAIOCCHI, Gianpaolo; HELLER, Patrick; SILVA, Marcelo Kunrath. Bootstrapping Democracy Stanford, CA: Stanford University Press, 2011.

BESLEY, Timothy; KUDAMATSU, Masayuki. "Health and democracy." The American Economic Review 96:2 (May): 313-318, 2006.

BOIX, Carles. "Democracy, development, and the public sector." American Journal of Political Science 45:1 (January): 1-17, 2001.

BRASIL. MINISTÉRIO DA SAÚDE. "Portal da Saúde: Datasus.” 2015.Disponível em: (http://www2. datasus.gov.br/DATASUS/index.php?area=0205). Acesso em 01.01.2016.

BROWN, David; HUNTER, Wendy. "Democracy and human capital formation education spending in Latin America, 1980 to 1997." Comparative Political Studies 37:7 (September): 842-864, 2004.

CLEARY, Matthew. "Electoral competition, 
participation, and government responsiveness in Mexico." American Journal of Political Science 51:2 (April): 283-299, 2007.

CORNWALL, Andrea; COELHO, Vera Schatten. Spaces for change? Vol. 4. Zed Books, 2006.

DAHL, Robert. Polyarchy: Participation and Opposition. New Haven: Yale University Press, 1971.

DIAMOND, Larry. Developing democracy: Toward Consolidation. Johns Hopkins University Press, 1999.

DIENER Ed. et al. Well-Being for public policy. New York: Oxford University Press, 2009.

DOLLAR, David; KRAAY, Aart. Trade, growth, and poverty. World Bank, Development Research Group, Macroeconomics and Growth, 2001.

DRÈZE, Jean; SEN, Amartya. An Uncertain Glory. Princeton: Princeton University Press, 2013.

DRYZEK, John. Deliberative democracy and beyond. Oxford University Press on Demand, 2000.

EVANS, Peter; HELLER, Patrick. "Human Development, State Transformation, and the Politics of the Developmental State." In Oxford Handbook on Transformation of the State. New York: Oxford University Press, 691-714, 2015.

FERRAZ, Claudio; FINAN, Federico. "Electoral accountability and corruption: evidence from the audits of local governments." American Economic Review 101:4 (April): 1274-311, 2011.

FERREIRA, Francisco. "Distributions in Motion: Economic Growth, Inequality, and Poverty Dynamics." World Bank Policy Research Working Paper No. 5424, 2010.

FOX, Jonathan. "Social Accountability: what does the evidence really say?" World Development 72 (August): 346-361, 2015.

FREY, Bruno; STUTZER, Alois. Happiness and Economics. Princeton, N.J.: Princeton University Press, 2002.

FUNG, Archon; WRIGHT, Erik Olin. Deepening democracy. New York: Verso. 2003

GERRING, John. et.al. "Electoral Democracy and Human Development." V-Dem Institute Working Paper 9, 2015.

GIBSON, Edward. Boundary Control. New York: Cambridge University Press, 2013.

GRINDLE, Merilee. Going Local. Princeton: Princeton University Press, 2007.

GURZA LAVALLE, Adrian, VOIGT, Jessica \&
SERAFIM, Lizandra. "O que fazem os conselhos e quando o fazem? Padrões decisórios e o debate dos efeitos das instituições participativas". Dados Revista de Ciências Sociais, Rio de Janeiro, 59 (3): 609-650, 2016.

HAGOPIAN, Frances. Traditional Politics and Regime Change in Brazil. Cambridge: Cambridge University Press, 1996.

HUBER, Evelyne. "Options for Social Policy in Latin America: Neoliberal versus Social Democratic Models" In: Welfare States in Transition, ed. Gosta Esping-Andersen. UK:SAGE, 141-191,1996.

HUBER, Evelyne; STEPHENS, John. Democracy and the left. Chicago, IL: University of Chicago Press, 2012.

HUNTER, Wendy; SUGIYAMA, Natasha Borges. Transforming Subjects into Citizens: Insights from Brazil's Bolsa Família." Perspectives on Politics 12: 4 (December): 829-845, 2014.

INSTITUTO DE PESQUISA ECONÔMICA APLICADA - IPEA. Ipeadata. "Pobreza - Pessoas Pobres, Pessoas Indigentes". Disponível em: http:// www.ipeadata.gov.br Acesso em 01.04.2016.

INSTITUTO BRASILEIRO DE GEOGRAFIA E ESTATISTICA -IBGE. Perfil dos Municípios Brasileiros - 2013. Disponível em: https:// ww2.ibge.gov.br/home/estatistica/economia/ perfilmunic/2013/default.shtm. Acesso em: 15.07.2016.

KOHLI, Atul. State-directed development. Cambridge: Cambridge University Press, 2004.

LAKE, David; BAUM; Matthew. "The invisible hand of democracy: political control and the provision of public services." Comparative Political Studies 34: 6 (August): 587-621, 2011.

LINDERT, Kathy et.al. "The Nuts and Bolts of Brazil's Bolsa Família Program: Implementing Conditional Cash Transfers in a Decentralized Context." World Bank, SP Discussion Paper No. 0709, 2007.

LUSTIG, Nora, LOPEZ-CALVA, Luis; ORTIZJUAREZ, Eduardo. "Declining inequality in Latin America in the 2000s: the cases of Argentina, Brazil, and Mexico." World Development 44 (April): 129141, 2013.

MACINKO, James; GUANAIS Frederico; SOUZA, Maria de Fatima de. "Evaluation of the impact of the Family Health Program on infant mortality in Brazil, 1990-2002." Journal of Epidemiology and Community Health 60:1 (January): 13-19, 2006.

MARSHALL, Thomas. Citizenship and Social Class. Garden City, NY: Doubleday, 1950. 
MCGUIRE, James. Wealth, health, and democracy in East Asia and Latin America. New York: Cambridge University Press, 2010.

MCNULTY, Stephanie. Voice and vote: decentralization and participation in post-Fujimori Peru. Palo Alto: Stanford University Press, 2011.

NUSSBAUM, Martha. Creating capabilities. Cambridge: Harvard University Press, 2011.

O’DONNELL, Guillermo. "Horizontal accountability in new democracies." Journal of Democracy 9:3 (July): 112-126, 1998.

OSTROM, Elinor. "Crossing the Great Divide: Coproduction, Synergy, and Development." World Development 24: 6 (June): 1073-87, 1996.

PATEMAN, Carole. "Participatory democracy revisited." Perspectives on Politics 10:1: (March) 7-19, 2012.

PERUZZOTTI, Enrique; SMULOVITZ, Catalina. "Societal Accountability in Latin American." Journal of Democracy 11:4 (October): 147-158, 2000.

PIRES, Roberto; VAZ, Alexander. Participação social como método de governo? Um mapeamento das" interfaces socioestatais" nos programas federais. Working Paper No. 1707. Instituto de Pesquisa Econômica Aplicada (IPEA). 2012.

POGREBINSCHI, Thamy; SAMUELS, David. "The Impact of Participatory Democracy: Evidence from Brazil's National Public Policy Conferences." Comparative Politics 46:3 (April): 313-332, 2014.

PRZEWORSKI, Adam et.al. Democracy and Development: Political Institutions and Material Well Being in the World. New York: Cambridge University Press, 2000.

PUTNAM, Robert; LEONARDI, Robert; NANETTI, Raffaelai. Making Democracy Work. Princeton: Princeton University Press, 1994.

RAVALLION, Martin; CHEN, Shaohua. "China's (uneven) progress against poverty." Journal of Development Economics 82: 1 (January): 1-42, 2007.

RASELLA, Davide et. al. "Effect of a conditional cash transfer programme on childhood mortality: a nationwide analysis of Brazilian municipalities." The Lancet 382: 9886 (July): 57-64, 2013.

RODRIK, Dani. "Growth versus poverty reduction: a hollow debate." Finance and Development 37:4 (December): 8-9, 2000.

ROSS, Michael. "Is democracy good for the poor?" American Journal of Political Science 50:4 (October): 860-874, 2006.

RUESCHEMEYER, Dietrich, STEPHENS, Evelyne
Huber; STEPHENS, John D. Capitalist development and democracy. Cambridge: Cambridge University Press, 1992.

SEN, Amartya. Development as freedom. New York: Alfred A. Knopf, 1999.

SNYDER, Richard. "Scaling down: The subnational comparative method." Studies in Comparative International Development 36:1 (March): 93-110, 2001.

SOARES, Fábio Veras; RIBAS, Rafael Perez, OSÓRIO, Rafael Guerreiro. "Evaluating the Impact of Brazil's Bolsa Família: Cash Transfer Programs in Comparative Perspective." Latin American Research Review 45:2. 174-90, 2010.

SOMERS, Margaret. Genealogies of Citizenship. Cambridge: Cambridge University Press, 2008.

SUGIYAMA, Natasha B.; HUNTER, Wendy. "Whither Clientelism?" Comparative Politics 46:1 (October): 43-62, 2013.

SUGIYAMA, Natasha B.. Diffusion of Good Government. Notre Dame, IN: University of Notre Dame Press, 2012.

TARROW, Sidney. Power in Movement. New York: Cambridge University Press, 1998.

TENDLER, Judith. Good government in the Tropics. Johns Hopkins University Press, 1997.

TEICHMAN, Judith. "The World Bank and Policy Reform in Mexico and Argentina." Latin American Politics and Society 46:1 (April): 39-74, 2004.

TOUCHTON, Michael; WAMPLER, Brian. "Improving Social Well-Being Through New Democratic Institutions." Comparative Political Studies 47:10 (September): 1442-1469, 2014.

VIANA, Ana Luiza; DAL POZ, Mario Roberto dal. "A Reforma do Sistema de Saúde no Brasil e o Programa de Saúde da Família." PHYSIS: Revista Saúde Coletiva, Rio de Janeiro, 8:2: 11-42, 1998.

WAMPLER, Brian. Activating Democracy. Notre Dame: University of Notre Dame Press, 2015.

WAMPLER, Brian; SUGIYAMA, Natasha B.; TOUCHTON, Michael. Democracy at Work: Pathways to Well-Being in Brazil. New York: Cambridge University Press, 2020.

WEYLAND, Kurt. Democracy without Equity. Pittsburgh, PA: University of Pittsburgh Press, 1996.

WORLD BANK. "Gini Index.” World Bank Indicators. 2016. Disponível em: https://data.worldbank.org/ indicator/SI.POV.GINI. Acesso em 15.07.2016.

WORLD HEALTH ORGANIZATION - WHO. Global Health Observatory (GHO) Data: Neonatal Mortality. 
World Health Organization. Disponível em: http:// www.who.int/gho/child_health/mortality/neonatal/ en. Acesso em 15.07.2016.

YASHAR, Deborah. Contesting Citizenship in Latin America. New York: Cambridge University Press, 2005.

XUE, Jinjun. Growth with Inequality. World Scientific Press. Singapore, 2012. 


\section{DEMOCRACY AR WORK: moving beyond elections to improve well-being}

\author{
Michael Touchton \\ Natasha Borges Sugiyama \\ Brian Wampler
}

\section{DÉMOCRATIE EN ACTION: aller au-delà des élections pour améliorer le bien-être}

\author{
Michael Touchton \\ Natasha Borges Sugiyama \\ Brian Wampler
}

How does democracy work to improve well-being? In this paper, we disentangle the component parts of democratic practice-elections, civic participation, expansion of social provisioning, local administrative capacity-to identify their relationship with well-being. Our analysis of an original dataset covering over 5,550 Brazilian municipalities demonstrates that competitive elections alone do not explain variation in infant mortality rates, one outcome associated with wellbeing. We move beyond elections to show how participatory institutions, social programs, and local state capacity can interact to buttress one another and reduce infant mortality rates. The result is a new understanding of how different aspects of democracy work together to improve a key feature of human development.
Comment la démocratie contribue-t-elle à améliorer le bien-être? Dans cet article, nous séparons les éléments constitutifs de la pratique démocratique - élections, participation sociale, expansion des programmes sociaux, capacité administrative municipale - pour identifier sa relation avec le bien-être. Notre analyse d'un ensemble de données original couvrant plus de 5550 municipalités brésiliennes montre que les élections compétitives n'expliquent pas à elles seules la variation des taux de mortalité infantile, résultat lié au bien-être. Nous allons au-delà des élections pour montrer comment les institutions participatives, les programmes sociaux et la capacité administrative municipale peuvent interagir pour se soutenir mutuellement et réduire les taux de mortalité infantile. Le résultat est une nouvelle compréhension de la façon dont différents aspects de la démocratie travaillent ensemble pour améliorer une caractéristique essentielle du développement humain.
Keywords: Democracy, Well-being, Participation, Social programs, Brazil.
Mots CLÉs: Démocratie, Bien-être, Participation, Programmes sociaux, Brésil.

Michael Touchton - Doutor em Ciência Política pela Universidade de Colorado, Boulder; professor do Departamento de Ciência Política, Universidade de Miami; líder docente em saúde global, Instituto de Estudos Avançados das Américas, desenvolvendo pesquisas na área de políticas públicas e saúde global. Suas mais recentes publicações são: Democracy at work: pathways to well-being in Brazil (Cambridge University Press) e Of governance and revenue: participatory institutions and tax compliance in Brazil (The World Bank).

Natasha Borges Sugiyama - Doutora em Governo pela Universidade de Texas em Austin; professora do Departamento de Ciência Política e Diretora do Centro de Estudos da América Latina e Caribe, Universidade de Wisconsin-Milwaukee; especialista em Políticas Sociais, Género e Desenvolvimento, e Cidadania no Brasil e na América Latina. Suas mais recentes publicações são: Democracy at work: pathways to well-being in Brazil (Cambridge University Press) e "Do CCTs Empower Women? Lessons from Brazil's Bolsa Família” (Latin American Politics and Society, vol. 62).

Brian Wampler - Doutor em Ciência Política pela Universidade de Texas, Austin; professor do Departamento de Ciência Política na Boise State Universty. Suas mais recentes publicações são Democracy at work: pathways to well-being in Brazil (Cambridge University Press) e "Designing Institutions to Improve Well-being: Participation, Deliberation, and Institutionalization” (European Journal of Political Research, vol. 58). 